\title{
Spatial Hypersurfaces in Causal Set Cosmology
}

\author{
Seth A. Major, ${ }^{1, *}$ David Rideout, ${ }^{2}, t$ and Sumati Surya ${ }^{3, \pm}$ \\ ${ }^{1}$ Department of Physics, Hamilton College, Clinton NY 13323 USA \\ ${ }^{2}$ Blackett Laboratory, Imperial College, London SW7 2AZ, UK \\ ${ }^{3}$ Raman Research Institute, CV Raman Avenue, \\ Sadashivanagar, Bangalore - 560 080, INDIA
}

(Dated: January 2006)

\begin{abstract}
Within the causal set approach to quantum gravity, a discrete analog of a spacelike region is a set of unrelated elements, or an "antichain". In the continuum approximation of the theory, a moment-of-time hypersurface is well represented by an "inextendible" antichain. We construct a richer structure corresponding to a "thickening" of this antichain containing non-trivial geometric and topological information. We find that covariant observables can be associated with such thickened antichains and transitions between them, in classical stochastic growth models of causal sets. This construction highlights the difference between the covariant measure on causal set cosmology and the standard sum-over-histories approach: the measure is assigned to completed histories rather than to histories on a restricted spacetime region. The resulting re-phrasing of the sum-over-histories may be fruitful in other approaches to quantum gravity.
\end{abstract}

\footnotetext{
*Electronic address: smajor@hamilton.edu

$\dagger$ Electronic address: d.rideout@imperial.ac.uk

${ }^{\ddagger}$ Electronic address: ssurya@rri.res.in
} 


\section{INTRODUCTION}

A major hindrance to the development of quantum gravity is the family of issues known as the problem of time [1]. Simply put, the problem is one of labeling states to record the evolution of physical quantities. In the canonical formulation, the problem of time arises because the Hamiltonian, which encodes the dynamics, is a constraint; since the observables commute with the constraints, they become "frozen". Thus, it appears that there are no dynamical observables in the theory [19]. In the path integral formulation, the problem arises both in finding a covariant way of expressing the initial and final states of geometry and in finding a measure such that the propagator sums over physically distinct configurations.

One approach to this problem is to abandon all reference to spatial geometries and instead consider only spacetime quantities. However, since much of classical and quantum physics is conceptualized in terms of moment-of-time and initial value formulations, it is important to know whether a covariant interpretation of spatial hypersurfaces is at all possible. The Hartle-Hawking no-boundary proposal, for example, provides one such interpretation within the framework of Euclidean sum-over-histories quantum gravity [3].

We examine this question within the causal set approach to quantum gravity. Our analysis suggests a general prescription for other sum-over-histories approaches to quantum gravity: instead of summing over histories between geometries at fixed times the measure is on "completed" histories containing transitions between the spatial hypersurfaces. This may be particularly relevant to the Causal Dynamical Triangulation (CDT) approach to quantising gravity in which one considers "sufficiently" completed histories [17].

In causal set quantum gravity, the underlying structure is a causal set $C$, which is a locally finite partial order [4, 5] : i.e., $C$ is a set with an order relation $\prec$ which is transitive $(x \prec y$ and $y \prec z \Rightarrow x \prec z)$, irreflexive $(x \nprec x)$ and locally finite $(|\operatorname{Past}(x) \cap \operatorname{Fut}(y)|<\infty)$ for any $x, y, z \in C$ (where Past $(y)=\{x \mid x \prec y\}, \operatorname{Fut}(y)=\{x \mid y \prec x\}$ and $|A|$ denotes set-cardinality.) The spacetime continuum arises as an approximation, rather than a limit of the theory, and quantities such as geometry and topology correspond to macroscopic degrees of freedom, analogous to thermodynamic quantities such as pressure and temperature for a gas of particles. While we expect a causal set to include both microscopic and macroscopic physical information, the latter should be well-described by physics in the continuum approximation.

A continuum manifold $(M, g)$ is said to approximate a causal set $C$ if and only if there 
exists a faithful embedding $\phi: C \rightarrow M$. The faithful embedding requires that the causal relations induced by the embedding agree with the order relation in $C$, and that the embedded points are sprinkled randomly via a Poisson process, at a spacetime density $\rho$, with high probability. A causal set embedded in a spacetime $(M, g)$ thus resembles a random spacetime lattice, where a link between two points implies a causal relation. In this lattice, a set of unrelated elements corresponds to a set of mutually spacelike events.

In the continuum, we define a moment-of-time surface (MoTS) to be a spacelike, acausal, edgeless hypersurface such that any other spacetime event is either to its causal future or past. This definition captures our requirement that the spatial hypersurface represent a global moment-of-time. An example of a MoTS is a Cauchy hypersurface in a globally hyperbolic spacetime. In the discrete context of causal sets, an antichain is a set of unrelated elements and corresponds, in an embedding, to a set of spacelike events. Thus, a natural analog of a MoTS is an inextendible antichain, i.e. an antichain such that any element not in it is related to it [20].

How good is this analogy? Given a faithful embedding $\phi: C \rightarrow M$, if $A$ is an inextendible antichain in $C$, there exist an uncountable infinity of MoTSs which contain $\phi(A)$. To what degree do they differ? Let $\Sigma_{1,2}$ be two such MoTSs. Consider any pair of points $p_{1,2}$ on $\Sigma_{1,2}$ for which the spacetime interval $I^{+}\left(p_{1}\right) \cap I^{-}\left(p_{2}\right)$ is non-empty (where $I^{ \pm}(x)$ represents the chronological future/past of $x)$. Since $\phi(A)$ is inextendible any such interval does not intersect $\phi(C)$. The probability for this to have happened via a Poisson sprinkling is given by $\exp \left[-\rho \operatorname{vol}\left(I^{+}\left(p_{1}\right) \cap I^{-}\left(p_{2}\right)\right)\right]$, which is appreciable only for $\operatorname{vol}\left(I^{+}\left(p_{1}\right) \cap I^{-}\left(p_{2}\right)\right) \lesssim 1 / \rho$. Thus, with "high probability" $\phi(A)$ represents a MoTS up to $\rho^{-1}$ differences, i.e. a MoTS is a good approximation to $A$. The analogy with a MoTS is further strengthened by results in 10] in which we show that the homology of a MoTS can be recovered from an inextendible antichain which it contains. However arising from the Lorentz invariance of the sprinkled causet, a crucial difference persists. Unlike a MoTS, $\phi(A)$ need not "capture" all irreducible causal relations or links, i.e., if $x, y \in C$ such that $x \prec y, x \in \operatorname{Past}(A), y \in \operatorname{Fut}(A)$, and if there exists no $z \in A$ for which $x \prec z \prec y$. The existence of such "missing links" might lead to observational consequences.

Since an antichain is simply a collection of unrelated elements, it is characterized only by its cardinality, which is not enough information to record dimension and topology, much less geometry. We therefore need a richer structure to extract the relevant information contained 
in a MoTS from the causal set. Indeed, as shown in [7, 8, 9], the larger causal set contains geometric and topological information. One might expect that not all the information in the causal set is required for this purpose, but rather only an appropriately defined subcauset which is a neighbourhood of the inextendible antichain. Such a neighbourhood has a continuum interpretation as a "thickened" MoTS with the scale of thickening corresponding roughly to the resolution of time in physical measurements.

One particularly useful definition of this neighbourhood is a future or past volume thickening of the inextendible antichain, which can be used to recover the homological information of a spatial hypersurface [10]. An inextendible antichain $A$ is thickened by a volume $v$ by adjoining to $A$ the set of elements to its future(past) whose past(future) up to and including $A$ has cardinality less than or equal to $v$. We define a (future volume) thickened antichain to be

$$
A_{v}=\{x \mid x \in \operatorname{Fut}(A) \cup A \text { and }|\operatorname{Past}(x) \backslash \operatorname{Past}(A)| \leq v\},
$$

so that $A_{0}=A$ (see Fig. 1]) A past volume thickened antichain is similarly defined. For both future and past volume thickenings, $A_{v}$ is convex in the larger causal set $C$, i.e. if $x \prec z \prec y$ for any $x, y \in A_{v}$, then $z \in A_{v}$.

The question that is of interest to us here is whether the discrete analogs of a MoTS and transitions between them can be given a covariant meaning in causal set quantum gravity. In the standard sum-over-histories formulation, the Hartle-Hawking wavefunction for a spatial geometry $(\Sigma, q)$ is

$$
\psi(q ; \Sigma)=\sum_{M} \int \mathcal{D} g e^{-S_{E}(g)},
$$

where the sum is over manifolds $M$ with boundary $\Sigma$ and the path integral is over (Euclidean) histories $g$ with $\left.g\right|_{\Sigma}=q$. This amplitude $\psi(q ; \Sigma)$ cannot be interpreted as the amplitude for $(\Sigma, q)$ to be a MoTS, since this requires that all subsequent events be to its causal future; only completed spacetime histories contain such subsequent information. To implement this within Lorentzian quantum gravity one requires the subclass $\Gamma \subset\{(M, g)\}$ of the set of "completed" histories containing $(\Sigma, q)$ as a MoTS. The amplitude is then $\psi(q ; \Sigma)=$ $\sum_{M} \int_{g} \mathcal{D} g e^{i S[g]}$, where $(M, g) \in \Gamma$.

Similarly, the transition amplitude between two spatial slices $\left(\Sigma_{1}, q_{1}\right)$ and $\left(\Sigma_{2}, q_{2}\right)$, cannot be interpreted as a transition amplitude between MoTSs. One must restrict to the subclass of completed histories $\Gamma_{12}$ such that for any $(M, g) \in \Gamma_{12}, \Sigma_{1}, \Sigma_{2}$ are non-intersecting MoTSs 


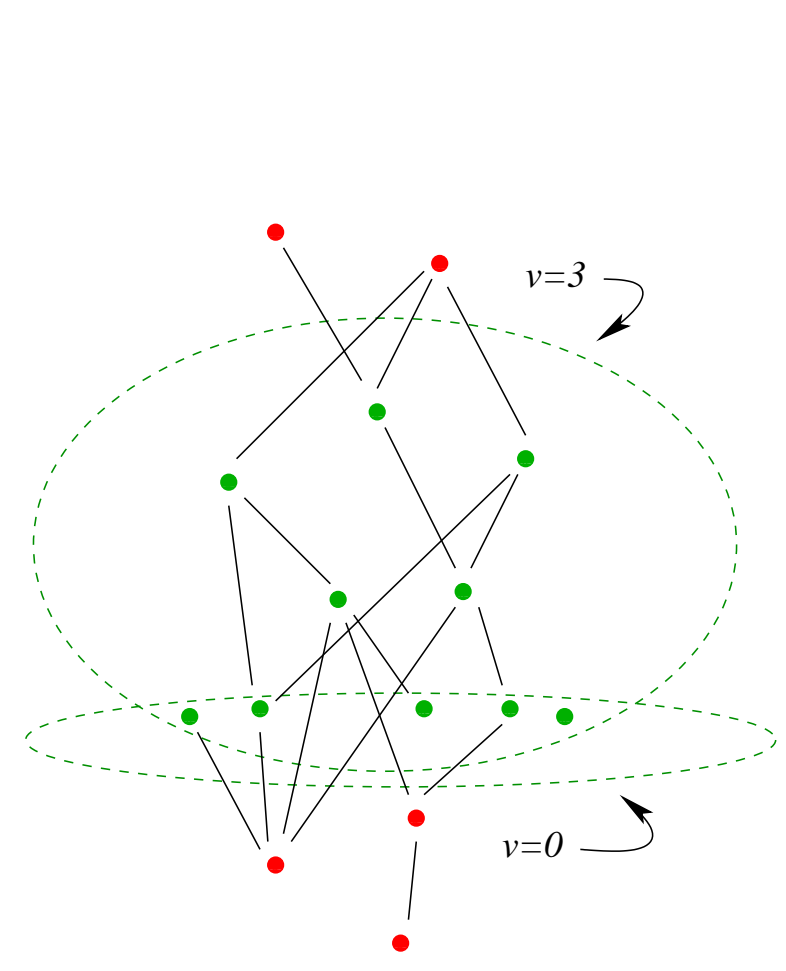

(a.)

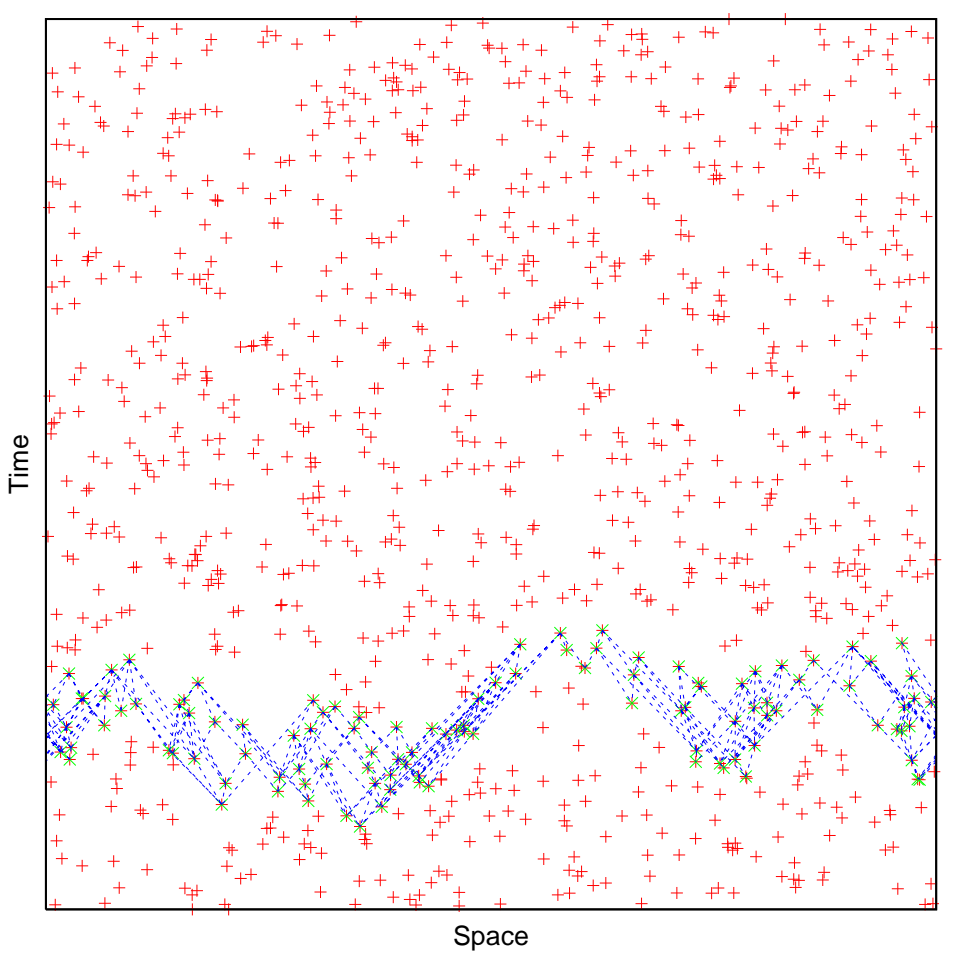

(b.)

FIG. 1: (a) An inextendible antichain in an abstract causal set is delineated by the $v=0$ dashed line. The larger $v=3$ thickened antichain is also delineated. Elements are shown as dots and links by solid lines. (b) A thickened antichain with $v=16$ is shown in a sprinkled causet embedded in $S^{1} \times I$. Elements in the sprinkling are denoted with a (red) "+" and elements in the thickened antichain are denoted with a (green) " $\times$ ". The causal relations in the thickened antichain are shown as (blue) dotted lines.

with $\Sigma_{1}$ to the past of $\Sigma_{2}$ and with the interpolating region between them either to the past or the future, but not both, of any other event in the spacetime. The transition amplitude between the two MoTSs is then $\sum_{M} \int_{g} \mathcal{D} g e^{i S[g]}$, with $(M, g) \in \Gamma_{12}$. This transition amplitude doesn't depend merely on the action of the interpolating region but also on the measure of the set of completed histories $\Gamma_{12}$ which contains such a region. This is a non-local prescription and differs significantly from that in the standard sum-over-histories approach.

Although this prescription is natural in the causal set context, it also provides a new framework for covariant sum-over-histories which may be useful in other approaches to quantum gravity. In the remainder of this article we briefly review the role of observables in 
causal set "classical sequential growth" dynamics in the next section. In section 【II we show that the existence of thickened antichains, and transitions between them, is measurable. They are observables. In the final section [V] we related these measures to more familiar conditional probabilities and reflect on how these ideas might be applied in the context of causal dynamical triangulations.

\section{OBSERVABLES IN CLASSICAL SEQUENTIAL GROWTH}

In the absence of a clear formulation of a quantum causal set dynamics, we turn to the classical stochastic growth (CSG) models of causets [11, 12] to flesh out the details of our proposal. Covariant observables in the class of (extended) generalised percolation models have been completely characterised [13, 14] and the hope is that they will provide an insight into the nature of observables in the quantum theory. We use the formalism developed in 13. to show that the causal set analogue of MoTSs and transitions between them can be assigned a covariant meaning in these theories.

In 13] the set of observables for a class of CSG models, called generalised percolation dynamics, was completely characterised in terms of covariant past sets or "stem sets". These results were recently generalised to the class of extended percolation dynamics [12] in [14]. More specifically, one can express the causal set dynamics in terms of a measure space, which is a triad $(\Omega, \mathcal{R}, \mu)$, with $\Omega$ the set of completed unlabeled causets being the sample space, $\mathcal{R}$ a $\sigma$-algebra on $\Omega$, and $\mu$ the probability measure obtained from the dynamics. A covariant observable of the theory then corresponds to a measurable physical question, i.e., an element of $\mathcal{R}$.

A subcauset $S$ of a causal set is called a stem if it is its own inclusive past, i.e., $S=$ $\operatorname{Past}(S) \cup S$. A stem set associated with a (finite) causet $S$ is the set of completed causal sets $C$ which contain $S$ as stem. The main result of [13, 14] was to show that, save sets of measure zero, the set of stem sets generates the $\sigma$-algebra $\mathcal{R}$. Moreover, since stems are past sets, the physical interpretation of these observables is straightforward. What we will prove is that thickened antichains and transitions between them admit a covariant, measurable interpretation in terms of stem sets and hence are elements of $\mathcal{R}$.

Because of the formulation of the dynamics as a sequential growth process, we need to impose the slightly stronger condition of past finiteness on the causets: $C$ is past finite if 
$|\operatorname{Past}(x)|$ is finite $\forall x \in C$. Let $\Omega(\mathbb{N})$ be the set of finite causets. A finite causet $E$ is a future end of a finite causet $C$ if $E$ is a subcauset of $C$ such that $E$ contains its own future in $C$. The past end is defined analogously and is equivalent to a stem. A future end $E$ of $C$ is said to be maximal if for every $e \notin E, e \prec E$. A maximal past end is similarly defined. A finite causet $I$ is said to interpolate between the subcausets $A$ and $B$ if $I$ has a maximal past end isomorphic to $A$, and a maximal future end of isomorphic to $B$, both of which are thickened antichains in $I$ and are disjoint in $I$. A causet $C$ contains an interpolating region $I$ with maximal ends $\{A, B\}$ if $I$ is a finite convex subcauset of $C$ interpolating between a maximal future and past end isomorphic to $A$ and $B$, respectively, which are both thickened antichains in $C$.

In the CSG models, one grows a causal set stage by stage, starting from a single element. Following the terminology of that paper, a finite causal set $P \in \Omega(n)$ is an ancestor of some $D \in \Omega(\mathbb{N}) \sqcup \Omega$ if $P$ is a stem in $D$. Conversely, $D$ is said to be a descendant of $P$. One obtains $D$ from $P$ by adding elements to $P$ maximally: starting from $P$, a single maximal element is added to $P$ to obtain the causal set $P^{\prime}$, and so on to get $D$. The new elements are maximal at the stage at which they are added, but do not need to remain maximal at subsequent stages.

For simplicity, we will confine our analysis to thickened antichains of finite cardinality and interpolators between such thickened antichains of fixed finite cardinality. A finite thickened antichain can be given the continuum interpretation of a "volume thickened" MoTS which has finite spacetime volume. Likewise, fixing the cardinality of the interpolator between a pair of finite thickened antichains corresponds to fixing the volume of the spacetime between two MoTSs, as in the unimodular approach to quantum gravity [15].

\section{OBSERVABLES, THICKENED ANTICHAINS, AND TRANSITIONS}

Let $\Phi_{A}$ denote the set of (past finite) completed causets containing the finite future or past volume thickening $A$ of an inextendible antichain $a$. Given a pair of finite causets $\{A, B\}$ containing inextendible antichains $a$ and $b$ respectively, and an $N \in \mathbb{N}$, let $\Phi_{A B}^{N}$ denote the set of completed causets containing any interpolator $I \in \Omega(N)$ with ends $\{A, B\}$ as an interpolating region. Assuming throughout that $A, B$ are non-empty, we show that:

Proposition: (i) $\Phi_{A}$ and (ii) $\Phi_{A B}^{N}$ are elements of the $\sigma$-algebra $\mathcal{R}(S)$ generated by the set 
of stem sets. They are therefore measurable sets in the generalised and extended percolation dynamics of $C S G$.

Proof: Given the result of [13, 14], it suffices to demonstrate that the sets $\Phi_{A}$ and $\Phi_{A B}^{N}$ lie in the $\sigma$-algebra generated by stem sets $\mathcal{R}(S)$.

(i) Let $\mathcal{Q}$ be the set of finite causets with $A$ as a maximal future end. For any $P \in \mathcal{Q}$, let $\left\{A_{i}\right\}$ be the (finite) set of isomorphic copies of $A$ contained in $P$ as a maximal future end, with each $A_{i}$ containing an inextendible antichain $a_{i}$ isomorphic to $a$. Let $S_{P}$ be the set of finite descendants of $P$ which do not contain any of the $A_{i}$ as a thickened antichain. Define the sets

$$
\Xi_{P} \equiv \bigcup_{D \in S_{P}} \operatorname{stem}(D), \Phi_{P} \equiv \operatorname{stem}(P) \backslash \Xi_{P}, \Phi \equiv \bigcup_{P \in \mathcal{Q}} \Phi_{P}
$$

Clearly, $\Phi \in \mathcal{R}(S)$, since $\mathcal{Q}$ is countable, and $\Xi_{p}$ is a countable union of stem sets for any $P \in \mathcal{Q}$. We now show that $\Phi \subseteq \Phi_{A}$. Assume otherwise, i.e., $\exists C \in \Phi$ which does not contain a subcauset isomorphic to $A$ as a thickened antichain. Since $C \in \Phi, \exists P \in \mathcal{Q}$ such that $C \in \operatorname{stem}(P) . \quad P$ contains a set of isomorphic copies $\left\{A_{i}\right\}$ of $A$ as maximal future ends, with inextendible antichains $a_{i}$, none of which are thickened antichains in $C$. Since $C \in \operatorname{stem}(P)$, the $A_{i}$ are convex in $C$ and the $a_{i}$ are antichains in $C$. Thus the only option then is that none of the $a_{i}$ are inextendible antichains in $C$. Thus, $C$ contains a finite stem $D \supset P$ for which this is true, so that $D \in S_{P}$. But since $C \in \operatorname{stem}(D), C \in \Xi_{P}$ or $C \in\left(\Phi_{P}\right)^{c}$. This must be true for all $P \in \mathcal{Q}_{C}$ where $\mathcal{Q}_{C}=\{P \in Q \mid C \in \operatorname{stem}(P)\}$. Thus, $C \in \bigcap_{P \in Q_{C}}\left(\Phi_{P}\right)^{c}=\left(\bigcup_{P \in Q_{C}} \Phi_{P}\right)^{c}$. Since for $P \in \mathcal{Q} \backslash \mathcal{Q}_{C}, C \notin \operatorname{stem}(P)$, this means that $C \notin \Phi$, which is a contradiction. Next, we show that $\Phi_{A} \subseteq \Phi$. Let $C \in \Phi_{A}$ and take $P$ to be the inclusive past of a subcauset $A$ in $C$ which occurs as a thickened antichain. $P$ is therefore a stem in $C$, so that $C \in \operatorname{stem}(P)$. Since $A$ is a thickened antichain in $C$, there exists no stem $D$ in $C$ containing $P$ for which the $a$ in $A$ is not an inextendible antichain. Neither can the convexity of $A$ be spoiled by any descendant. Thus $C \notin \Xi_{P}$, and hence $C \in \Phi$.

Note: The measurability of the set of past finite causets containing a "post", or an element related to every other element in the causet, is a special case, when $A$ is a single element antichain. 
(ii) The measurability of $\Phi_{A B}^{N}$ follows similarly. There exist a finite set $\Psi$ of interpolators $I \in \Omega(N)$ with ends $\{A, B\}$. Let $\mathcal{Q}$ be the finite set of causets which contain any $I_{i} \in \Psi$ as an interpolating region and as a maximal future end. For any $P \in \mathcal{Q}$, let $\left\{I_{i}\right\}$ be the (finite number of) $I$ 's $\in \Psi$ contained in $P$ as an interpolating region and as a maximal future end, and let the ends of each $I_{i}$ be $\left\{A_{i k}, B_{i l}\right\}$, where $A_{i k}, B_{i l}$ are isomorphic to $A$ and $B$, respectively, with $a_{i k}, b_{i l}$ the respective inextendible antichains isomorphic to $a, b$. Let $S_{P}$ be the set of descendants of $P$ which do not contain any copy of any of the $I_{i} \subset P$ as an interpolating region. We may then define the set $\Phi \in \mathcal{R}(S)$ as in (i). The proof is now logically identical to that of (i).

The proof above can be simply modified to accommodate more generally defined thickened antichains. For example, a thickened antichain $A$ can be defined to be a convex subcauset of $C$ which contains any antichain $a \subset A$ inextendible in $C$. Or, it could be defined to be convex and "separating" in $C$, the latter term meaning that every element of $C$ not in $A$ is related to an element of $A$.

\section{DISCUSSION}

As is obvious from our construction, the measure of $\Phi_{A}$ is the probability that the thickened antichain $A$ appears at least once in a causal set. For example, if the measure of $\Phi_{A}$ is one, then this means that all completed causets that can occur in the dynamics contain $A$ as a thickened antichain, and if it is zero, then none of the causets can contain $A$ as a thickened antichain. Similarly, the measure of $\Phi_{A B}^{N}$ is the probability that an interpolating region of cardinality $N$ with the ends $A$ and $B$ appears at least once in a causet. This provides a covariant definition of transitions between discrete analogs of MoTSs. Relating the measure $\mu\left(\Phi_{A B}^{N}\right)$ on $\Phi_{A B}^{N}$ to a transition probability, however, is a more subtle issue since it is the conditional probability for the transition from $A$ to $B$ given that $A$ occurs [21]. If we merely divide $\mu\left(\Phi_{A B}^{N}\right)$ by the measure $\mu\left(\Phi_{A}\right)$ on $\Phi_{A}$, then this gives us the probability that a causet will contain at least one interpolating region from $A$ to $B$, given that $A$ occurs. This differs from the standard interpretation, since it leaves open the possibility that other $A$ 's in a causet may not transition to $B$ with the same probability.

The measure on $\Phi_{A}$ can be used to determine the measure on spatial topologies in the following sense: spatial topologies corresponding to $A$ are precluded if $\Phi_{A}$ has vanishingly 
small probability measure. Likewise, the occurrence of transitions from $A$ to $B$ are precluded if $\Phi_{A B}$ has vanishingly small probability. Restricting to a "cosmic time" $T$, which we take to be the cardinality of the inclusive past, is also useful for cosmological predictions, since we can ask what is the probability for $A$ to occur at some suitable $T$. On classical scales, one doesn't expect to see topology change [16]. Hence, if $T$ is chosen to be large enough so that topology change can no longer occur, then this would amount to a prediction for the spatial topology of the universe, in the event that our analysis survives quantisation.

We have used the CSG model to test our hypothesis that thickened antichains and transitions between them can be given a covariant interpretation in causal set dynamics. Moreover, we suggest that the continuum approximation of a thickened antichain is a thickened MoTS which contains the geometric and topological information of a MoTS. This provides us with a new covariant understanding of spatial hypersurfaces and transitions between them. Indeed, if the observability of stem sets survives quantisation, our analysis and interpretation would extend trivially to the quantum picture.

What is new - and may have relevance outside the context of causal sets - is that we have defined a covariant procedure for identifying a sub-region of the universe, independent of a choice of coordinate time. This notion of a covariant sum-over-histories may be useful in other approaches to quantum gravity in computing transitions over a finite spacetime volume and on completed histories. In CDT, since the histories are sufficiently completed, the probabilities can be obtained by performing a search in the space of causal triangulations for a particular three geometry $A$, or for a triangulated region of volume $V$ containing $A$ and $B$ as initial and final boundaries. Here $A$ and $B$ do not necessarily have to be individual geometries, but could be required to be members of sets of geometries which posses some property. The notion of a thickened antichain also finds a resonance in CDT. In [17] it was shown that the spectral dimension for spatial hypersurfaces is 1.5 rather than 3 in 4 dimensional spacetimes and that thickening by a single unit gives a value of a little over 2 . Our work on the homology of thickened antichains [10] suggests that the spatial hypersurface needs to be thickened to a scale much larger than the discreteness scale before it can correctly reproduce continuum topological information, including dimension.

Causal sets also make their appearance in the spin foam framework, as causal spin foams (CSF) 18]. Our prescription suggests a way to obtain a spatial hypersurface in an abstract CSF, where again, the thickening roughly corresponds to a time-measurement. To the 
extent that the CSF approach is a sum-over-histories formulation of quantum gravity, our construction suggests a way of obtaining covariant (spacetime) observables in this approach.

We would like to thank Fay Dowker and Joe Henson for discussions. This work was supported in part by a Research Corporation grant to SAM.

[1] C.J. Isham, "Canonical Quantum Gravity and the Problem of Time", L. A. Ibort and M. A. Rodriguez (eds.), Integrable Systems, Quantum Groups, and Quantum Field Theories (Kluwer Academic Publishers, London, 1993) pp. 157-288 arXiv:gr-qc/9210011; K.V. Kuchař, "Time and Interpretations of Quantum Gravity", G. Kunstatter, D. Vincent and J. Williams (eds.), Proceedings of the $4^{\text {th }}$ Canadian Conference on General Relativity and Relativistic Astrophysics (World Scientific, 1992).

[2] C. Rovelli, Phys.Rev. D 65, 124013 (2002).

[3] J. B. Hartle and S. W. Hawking, Phys. Rev. D 28, 2960 (1983).

[4] L. Bombelli, J. H. Lee, D. Meyer and R. Sorkin, Phys. Rev. Lett. 59, 521 (1987).

[5] R. D. Sorkin, "Causal sets: Discrete gravity (Notes for the Valdivia Summer School)" arXiv:gr-qc/0309009.

[6] R. D. Sorkin, Int. J. Theor. Phys. 39, 1731 (2000).

[7] R. P. Stanley, Enumerative Combinatorics: Volume I (Wadsworth and Brooks/Cole, Monterey, California, 1997).

[8] R. D. Sorkin, Int. J. Theor. Phys. 30, 923 (1991).

[9] G. Brightwell and R. Gregory, Phys. Rev. Lett. 66, 260 (1991).

[10] S. A. Major, D. P. Rideout, and S. Surya, "On recovering continuum topology from a causal set", in preparation.

[11] D. P. Rideout and R. D. Sorkin, Phys. Rev. D 61, 024002 (2000) arXiv:gr-qc/9904062.

[12] M. Varadarajan and D. Rideout, "A general solution for classical sequential growth dynamics of causal sets" arXiv:gr-qc/0504066.

[13] G. Brightwell, H. F. Dowker, R. S. Garcia, J. Henson and R. D. Sorkin, Phys. Rev. D 67, 084031 (2003); arXiv:gr-qc/0210061 G. Brightwell, H. F. Dowker, R. S. Garcia, J. Henson 
and R. D. Sorkin, "General covariance and the 'problem of time' in a discrete cosmology" arXiv:gr-qc/0202097.

[14] F. Dowker and S. Surya, "Observables in extended percolation models of causal set cosmology" arXiv:gr-qc/0504069.

[15] R. D. Sorkin, "A Modified Sum-Over-Histories for Gravity", B.R. Iyer, A. Kembhavi, C.V. Vishveshwara (eds.), Highlights in Gravitation and Cosmology (Cambridge University Press, Cambridge, 1988); R. D. Sorkin, Framework For Gravity,” Int. J. Theor. Phys. 33, 523 (1994); W. G. Unruh, Gravity," Phys. Rev. D 40, 1048 (1989).

[16] R. P. Geroch. J. Math. Phys, 8:782, 1967; R. D. Sorkin, Phys. Rev. D 33, 978 (1986).

[17] J. Ambjorn, J. Jurkiewicz and R. Loll, Phys. Rev. D 72, 064014 (2005).

[18] F. Markopoulou, L. Smolin, Nucl.Phys. B508 409 (1997).

[19] However, observables may be expressed as correlations between dynamical degrees of freedom. See 2] and references therein.

[20] An analogy between a spatial hypersurface and an inextendible antichain (often called a maximal antichain) is suggested in [6]. We clarify that the appropriate continuum analog to an inextendible antichain is a MoTS.

[21] We thank Joe Henson for pointing this out. 